\title{
Design and realization of flexible droplet-based lab-on-a-chip devices
}

\section{From theory to practice}

\author{
G. Fink, M. Hamidović, A. Springer, R. Wille, W. Haselmayr
}

\begin{abstract}
This article provides an overview on the emerging field of droplet-based microfluidic networks. In such networks, droplets i.e., encapsulating biochemical samples can be adaptively transported via microchannels through different operations for particular experiments. This approach is particularly promising for the next generation of lab-on-a-chip devices, which should support more complex operations and more flexibility. We give an accessible introduction to droplet-based microfluidics and describe the principles, of microfluidic switches, which are the main components in microfluidic networks. Based on these principles we present the addressing schemes for microfluidic bus networks. Since the design of microfluidic networks is a rather complex task, which requires the consideration of a huge number of physical parameters, we introduce design automation methods and simulation tools. Finally, we present a method for the precise generation of individual droplets, which enables the practical realization of microfluidic networks. Moreover, we show the latest experimental results on droplet generation and switching
\end{abstract}

Keywords: droplet-based microfluidics; lab-on-a-chip; microfluidic networks

\section{Design und Realisierung von flexiblen tröpfchenbasierten Chiplaboren: Von der Theory zur Praxis.}

Dieser Artikel gibt einen Überblick über das Forschungsfeld der mikrofluidischen Netzwerke. In solchen Netzwerken werden Tröpfchen (die bspw. biochemische Proben enthalten) adaptiv durch verschiedene Prozessstufen über Mikrometer-dünne Kanäle transportiert, um bestimmte Untersuchungen durchzuführen. Dieser neue Ansatz ist vor allem für die nächste Generation an Chiplaboren (engl. lab-on-a-chip) sehr vielversprechend, welche komplexere Operationen und mehr Flexibilität unterstützen sollen. Wir besprechen die Grundlagen tröpfchenbasierter Mikrofluidik und beschreiben die Prinzipien mikrofluidischer Schalter (engl. switch), welche die Hauptkomponenten in mikrofluidischen Netzwerken darstellen. Basierend auf diesen Prinzipien stellen wird die Adressierungsschemas in mikrofluidischen Busnetzwerken vor. Da der Entwurf von mikrofluidischen Netzwerken eine komplexe Aufgabe ist, bei der eine große Anzahl von physikalischen Parametern berücksichtigt werden muss, stellen wir verschiedene Ansätze zur Entwurfsautomatisierung und Simulation vor. Schließlich präsentieren wir eine Methode zur präzisen Erzeugung von Tröpfchen, die eine praktische Realisierung von mikrofluidischen Netzwerken ermöglichen soll. Weiters zeigen wir die neuesten experimentellen Ergebnisse zur Tröpfchengenerierung bzw. zur Realisierung von Schaltern.

Schlüsselwörter: tröpfchenbasierte Mikrofluidik; Chiplabor; mikrofluidische Netzwerke

Received January 31, 2020, accepted February 28, 2020, published online March 17, 2020

(c) The Author(s) 2020

\section{Introduction}

Microfluidics deals with the control and manipulation of small amounts of fluids (in the order of few micro- to pico-liters) and provides technological advances to the life sciences. It is an enabling technology when it comes to, e.g., biological cell studies, high throughput drug development, and diagnostic screenings. Corresponding devices are called Lab-on-a-Chip (LoC), which minimize, integrate, automate, and parallelize typical laboratory operations such as mixing, heating, incubation, etc. on a single chip $[9,31]$. Especially, the platform where droplets are used to confine certain samples allows to conduct a broad range of chemical and biochemical experiments [21,34]. In this platform, such droplets flow through closed microchannels inside a second immiscible fluid which acts as a carrier for the droplets. This has several advantages compared to conventional methods, since it requires significantly less reagent and sample volumes (e.g. relevant for restricted samples or costly reagents), facilitates shorter reaction times and, hence, higher throughput of numerous laboratory activities [34].
However, most droplet-based microfluidic devices process the available operations in a pre-defined way [34]. This obviously limits its flexibility. Hence, in order to allow for a more flexible use, the concept of droplet-based microfluidic networks has been proposed [7]. This allows to route cargo droplets, encapsulating biochemical samples, through different sequences of modules that process different operations in an order which is not pre-defined but rather can be adjusted for a particular application and/or experiment.

Fink, Gerold, Institute for Integrated Circuits, Johannes Kepler University, Altenberger Str. 69, 4040 Linz, Austria (E-mail: gerold.fink@jku.at); Hamidović, Medina, Institute for Communications Engineering and RF-Systems, Altenberger Str. 69, 4040 Linz, Austria (E-mail: medina.hamidovic@jku.at); Springer, Andreas, Institute for Communications Engineering and RF-Systems, Altenberger Str. 69, 4040 Linz, Austria

(E-mail: andreas.springer@jku.at); Wille, Robert, Institute for Integrated Circuits, Johannes Kepler University, Altenberger Str. 69, 4040 Linz, Austria (E-mail: robert.wille@jku.at); Haselmayr, Werner, Institute for Communications Engineering and RF-Systems, Altenberger Str. 69, 4040 Linz, Austria (E-mail: werner.haselmayr@jku.at) 


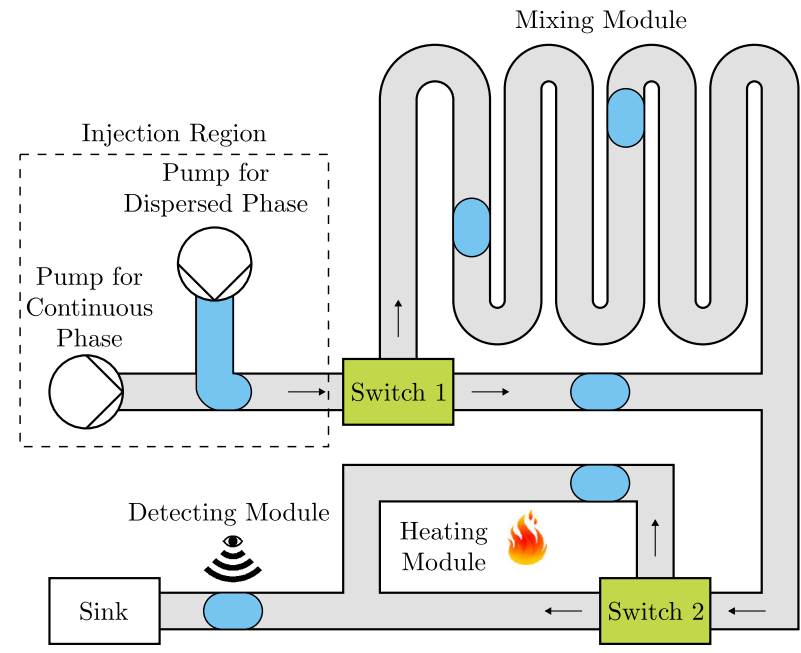

Fig. 1. Simple example of a droplet-based microfluidic network

As an example, a simple microfluidic network with some typical components is sketched in Fig. 1. In the injection region, pumps produce a flow of two immiscible fluids, the so-called continuous and dispersed phase. When both phases meet, e.g., in a T-junction, droplets are formed [21] and injected into the network, while the continuous phase acts as a carrier for the droplets. ${ }^{1}$ After the injection, the droplets flow towards different modules, e.g., a mixing module, a heating module, and a detecting module, which are all used to perform different operations. More precisely, the mixing module is used to mix the reagents inside a droplet, the heating module increases the temperature of a droplet (triggering a reaction of the samples inside the droplet), and the detecting module is used to screen a droplet in order to evaluate the reaction of the reagents.

In order to control if a corresponding droplet should be routed towards a module or not, microfluidic switches can be utilized. Such a switch is able to control the path of a droplet and only exploits passive hydrodynamic effects, ${ }^{2}$ i.e., the switch does not use active and costly components such as valves. This way the concepts of droplet-based microfluidic networks allow the realization of various experiments and applications.

However, the process from the conceptual design to an actually working realization of a microfluidic network is rather complex and can quickly become very expensive in terms of time as well as financial costs. More precisely, generating an initial design, validating the design's functionality, fabricating the resulting microfluidic network, and finally operating the network so that it is working as intended are all difficult tasks that require dedicated expertise and, ideally, tool support.

In this paper, we provide an overview on the respectively needed steps and how they can be mastered. To this end, we first review the basics and the underlying model in Sect. 2. Afterwards, the core components-namely the switches which control whether droplets are routed to modules or not-as well as the correspondingly resulting networks are described in Sect. 3. Using these concepts, microfluidic networks can be designed and fabricated. How automatic

\footnotetext{
${ }^{1}$ The precise generation of droplets is discussed in detail in Sect. 5 .

2 Please note that there exist various other approaches for the droplet control, such as electrical field [33] and acoustic waves [36]. However, such systems require a complex fabrication process and are not biocompatible in some settings.
}

methods for the design and simulation may help here and how the fabrication process actually can be conducted is then described in Sect. 4 and Sect. 5, respectively. All descriptions are thereby kept brief, but references for further reading are provided. Finally, the paper is concluded in Sect. 6.

\section{Basics}

In order to describe the behavior of microfluidic networks and develop corresponding design automation tools and simulation methods that aid designers during the design process, a proper physical model is needed. Here the one-dimensional (1D) analysis model proposed in [32] has become a suitable option. Although this abstract model does not cover all physical phenomena it has the advantage of low costs with respect to setup and computation time compared to, e.g., CFD simulations [13]. Because of this, the model can be used for deriving the specification of the design, for initially validating the functionality by using simulation, and also for design exploration. Hence, we cover this model in more detail in the following.

The 1D analysis model can be applied in scenarios, where a fully developed, laminar, viscous, and incompressible flow occurs, i.e., in scenarios with low Reynolds numbers. Microfluidic devices usually work at low Reynolds numbers ( $R e \ll 1)$, due to the small ratio of inertial to viscous forces-caused by the relatively small channel crosssections and flow rates. Moreover, this also allows to neglect inertial effects such as gravity, separation, secondary flow and turbulences.

As a result, the flow inside a microfluidic channel can be described by Hagen-Poiseuille's law [2, 32]

$$
\Delta P=Q R,
$$

where $Q$ is the volumetric flow rate, $\Delta P$ the pressure drop along the channel, and $R$ the hydrodynamic resistance of the channel. This hydrodynamic resistance depends on the channel geometry (i.e., length $I$, width $w$, and height $h$ ) as well as the dynamic viscosity of the continuous phase $\mu_{\mathrm{c}}$. For rectangular channels ${ }^{3}$ with a section ratio $h / W<1$, the hydrodynamic resistance is defined by [12]

$$
R=\frac{a \mu_{c} l}{w h^{3}}
$$

where a denotes a dimensionless parameter given by

$$
a=12\left[1-\frac{192 h}{\pi^{5} w} \tanh \left(\frac{\pi w}{2 h}\right)\right]^{-1} .
$$

Furthermore, when a droplet flows through a microfluidic channel, it causes an additional resistance inside this channel. As proposed in [14], a droplet with the length / Droplet increases the resistance of the segment it occupies inside the channel by

$$
R_{\text {Droplet }}=b \frac{a \mu_{c} l_{\text {Droplet }}}{w h^{3}}
$$

where $b$ is a factor which depends on the particular setup (used fluids, material, etc.) and usually lies between 2 and 5 .

Moreover, pumps produce a force at the input channels of a microfluidic network, which can be either a constant volumetric flow rate $Q_{\text {in }}$ (e.g. by using syringe pumps) or a constant pressure gradient $\Delta P_{\text {in }}$ (e.g. by using peristaltic pumps) and drives the continuous phase as well as the droplets inside the network.

Furthermore, a duality between Hagen-Poiseuille's law in the microfluidic domain and Ohm's law in the electrical domain exists [32]. In fact, all concepts and laws of the 1D model are equivalent to the

${ }^{3}$ The hydrodynamic resistance of channels with circular cross section is given by $R=\left(8 \mu_{c}\right) /\left(\pi r^{4}\right)$, with $r$ being the radius of the channel [2]. 

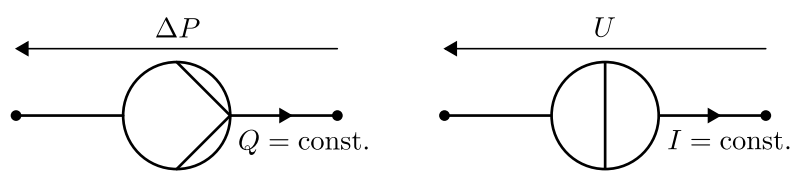

(a) Syringe Pump $\rightarrow$ Current Supply
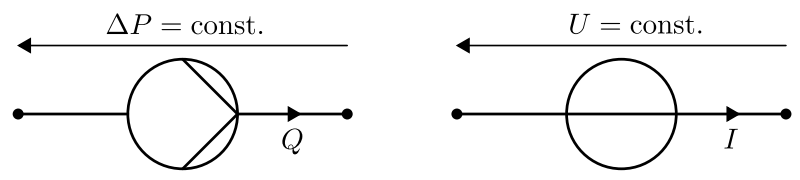

(b) Peristaltic Pump $\rightarrow$ Voltage Supply
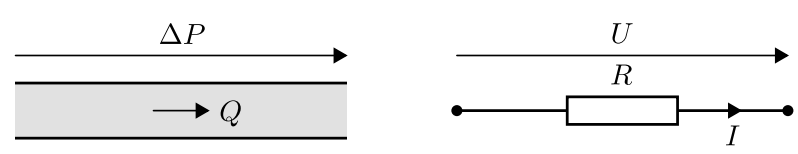

(c) Channel $\rightarrow$ Resistance
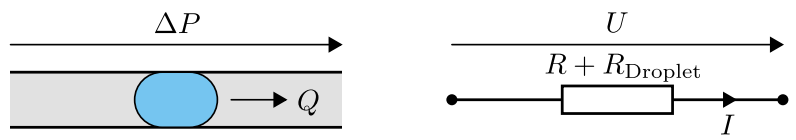

(d) Channel with Droplet $\rightarrow$ Combined Resistance

Fig. 2. Duality between microfluidic components (left-hand side) and electrical components (right-hand side)

corresponding laws for electrical circuits. This allows to apply well established methods and means from the electrical domain, such as Kirchoff's laws, which drastically enhance the process of designing and simulating microfluidic networks. Figure 2 illustrates this duality for the most basic components.

\section{Microfluidic networks}

Microfluidic networks refer to the interconnection of different microfluidic devices (e.g., heating or mixing module) using so-called microfluidic switches [7]. The aim of such networks is the realization of programmable, flexible, and biocompatible microfluidic systems. The droplets' path within the network is controlled by microfluidic switches that only exploit hydrodynamic effects and, thus, the main design parameters of microfluidic networks are the channel geometries and hydrodynamic forces (e.g., volumetric flow rate).

So far, two different types of microfluidic switches have been proposed: (i) single-droplet switches that control the path of a single droplet [8]; and (ii) multi-droplet switches that control the path of multiple droplets [3]. Moreover, various network topologies have been investigated: (i) application-specific [17]; (ii) ring [8]; and (iii) bus $[10,37]$ networks. In the following, due to lack of space, we will only provide a detailed discussion on the single-droplet switch and microfluidic bus networks. ${ }^{4}$

\subsection{Single-droplet switch}

The basic idea is to control the path of a cargo droplet (encapsulating for example biochemical samples) by a leading control droplet (containing no sample) that changes the state of the switch to steer the following cargo droplet along the desired channel. Following the terminology in classical network theory the control and cargo droplets are referred to as header and payload droplet, respectively.

${ }^{4}$ For more details on the multi-droplet switch and on different network architectures please refer to the literature cited above. Moreover, a comprehensive survey on droplet-based microfluidic networks is given in $[25,29,30]$.

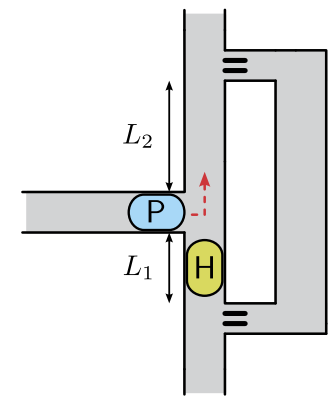

(a)

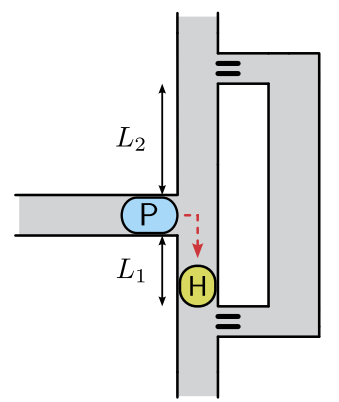

(b)
Fig. 3. Principle of size-based switching

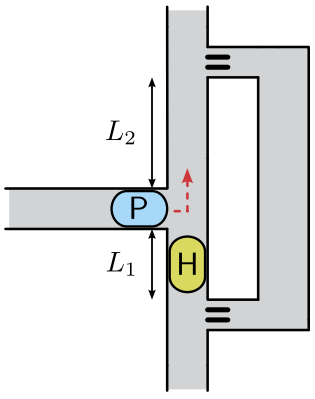

(a)

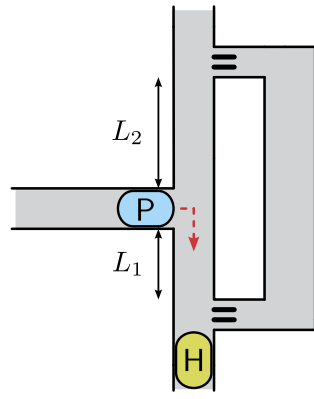

(b)
Fig. 4. Principle of distance-based switching

The single-droplet switch is shown in Figs. 3 and 4 and consists of a T-junction and two asymmetric channels 1 and 2 with a length of $L_{1}$ and $L_{2}$, respectively. It is assumed that $L_{1}<L_{2}$ and, thus, according to (2), the hydrodynamic resistance of channel 2 is higher than for channel 1. Moreover, the ends of channel 1 and 2 are connected by a so-called bypass channel. Such a channel has a much lower hydrodynamic resistance than channel 1 and 2 and it is designed in a way that droplets do not enter it while letting the continuous phase flow freely [6]. Hence, the pressure at the end of channel 1 and 2 is approximately the same and the volumetric flow rates in both channels are largely unaffected by the connected downstream circuits. In other words, the volumetric flow rates in the single-droplet switch only depend on the characteristics of channel 1 and 2 and on the possible presence of a droplet. Hence, the bypass channel is very important when multiple switches are cascaded.

When a header droplet with a following payload droplet flows towards the switch, the header droplet will always proceed along channel 1, due to its lower hydrodynamic resistance. The path followed by the payload droplet is determined by the changes of the volumetric flow rates produced by the header droplet. In particular, the switching mechanism can be controlled in two ways: (i) sizebased switching, exploiting the size of the header droplet [37] and; (ii) distance-based switching, using the distance between header and payload droplet [10].

For size-based switching the distance between header and payload droplet must be smaller than $L_{1}$ and, thus, the payload droplet reaches the junction before the header droplet has left channel 1. To steer the payload droplet into channel 2 the header droplet must be sufficiently large in order to increase the hydrodynamic resistance of channel 1 beyond that of channel 2 (see Fig. 3a). If this is not fulfilled the payload droplet will follow the header droplet along channel 1 (see Fig. 3b). 


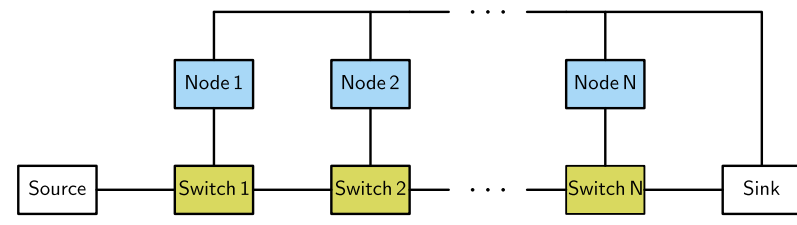

Fig. 5. Microfluidic bus network

For distance-based switching, the size of the header droplet is fixed and it increases the hydrodynamic resistance of channel 1 beyond that of channel 2 . To steer the payload into channel 2, its distance to the header droplet must be smaller than $L_{1}$, i.e. the header droplet is still in channel 1 (see Fig. 4a). On the other hand if the header droplet has already left the channel when the payload reaches the junction, it follows the header droplet into channel 1 (see Fig. 4b).

\subsection{Microfluidic bus network}

A microfluidic bus network consists of a main microfluidic channel (i.e., the bus) to which different microfluidic nodes (modules, cf. Sect. 1) are connected through microfluidic switches. In particular, the switches are cascaded, in which one outlet leads to the inlet of the next switch and the other outlet is connected to the associated network node. Moreover, the inlet of the first switch and the outlet of the last switch is connected to the droplet source and sink, respectively. The source is assumed to be able to generate different types of droplets at prescribed times with certain size and at a certain distance. ${ }^{5}$ The topology of a microfluidic bus network is depicted in Fig. 5.

For size-based switching (see Sect. 3.1) the address of the targeted network node is encoded in the header droplet size. Thus, the header droplet must increase the hydrodynamic resistance of channel 1 such that the payload droplet is steered towards the targeted node. On the other hand, the switching process must not be triggered for all previous switches. This can be accomplished by increasing the length of channel 2 for the upstream switches [37].

In distance-based switching (see Sect. 3.1), the distance between header and payload droplet determines the targeted network node. If the distance between the droplets is below the length of channel 1 , the payload droplet is deviated to the associated node. Otherwise, both droplets flow towards the next downstream switch and their distance is reduced. Thus, in order to avoid that the switching process is started before the targeted node, the distance at the source must be carefully selected taking the distance reduction into account.

Recently, a comprehensive analysis and comparison of both addressing schemes have been presented [24]. It has been shown that compared to size-based encoding, distance-based switching offers a simple and generic chip design (all switches have the same geometry), support larger networks, but suffers from low throughput.

\section{Automatic design and simulations}

While microfluidics is a prospective field which provides technological advances to the life sciences, the design and layout process of microfluidic devices is still in its infancy, and, thus, is mainly accomplished manually so far. This is particularly critical, since their design is a rather complex task where a huge number of physical parameters need to be considered. This frequently results in

${ }^{5}$ Please refer to Sect. 5 for the practical implementation of this assumption. a "trial-and-error" scheme, i.e., fabricating prototypes, observe their behavior, and refining the design until a working design is obtained - a time-consuming and rather costly process. In order to overcome this "trial-and-error" approach, design automation tools as well as simulation methods can be employed. These tools and simulation methods allow for deriving the design, for validating the functionality of the design, and for exploring alternative designs, without the need of an actual fabricated prototype. In the following, we provide a brief overview on some available tools and methods.

\subsection{Design automation}

As already mentioned above, the design process of microfluidic devices, is mainly conducted by hand-resulting in time consuming and labor-intensive processes. Design automation tools for microfluidic devices address this issue, by providing the designer with methods and means to automatically generate proper designs.

One example of such a tool is the Meander Designer proposed in [16], which aids designers in drawing meander channel structures for microfluidic devices. Meander channels are a central microfluidic component which are often integrated in many different platforms such as pressure driven, droplet-based, and paper-based microfluidics. However, even for this frequently re-occurring component, designers still have to manually draw the meander channel for their respective application and design rules. The Meander Designer addresses this issue and automates this tedious task, while still retaining the full control over the design. More precisely, the designer only has to provide corresponding parameters such as the desired hydrodynamic resistance, fabrication parameters, as well as the positions of the inlets and outlets. Based on that, a design satisfying these parameters is automatically generated.

In order to design a droplet-based microfluidic network, all used components have to be properly chosen, such as the pumps, modules, switches, and channels. The specifications of all components and how they are connected determine the flow state and, thus, the path of the droplets. Especially the specification of channels (i.e. their resistances) can be varied in a broad bandwidth and, by this, their dimensioning constitutes a significant challenge. Moreover, a huge number of constraints and dependencies have to be considered and already slight changes of a single channel may alter the behavior of the entire microfluidic system. In the work proposed in [19], these problems are addressed by introducing methods which automatically allow to validate whether a manually derived specification indeed works as intended, i.e. fulfills certain objectives, as well as conduct the dimensioning to obtain a proper specification. Moreover, the resulting designs afterwards can also be used to automatically generate proper droplet sequences [20] as well as to evaluate the robustness of the resulting design [11].

\subsection{Simulation}

In order to test the functionality and validate the design of a microfluidic network without the need of an actual fabricated prototype, tools that simulate the behavior of corresponding networks can be used.

One example of such a tool is the simulator proposed in [18], which utilizes the 1D model described in Sect. 2 and simulates droplet-based microfluidic devices. Since the analogy between the microfluidic and electrical domain exists, Kirchhoff's current and voltage laws can also be applied. These two laws allow to setup an equation system which can be used to determine the flow state (i.e. pressure drops and flow rates in all channels) of a microfluidic network. Based on this flow state the velocities of the droplets as well as the paths the droplets take inside the microfluidic network 
can be obtained. However, the flow state as well as the velocities of the droplets change as soon as (1) a new droplet is injected, (2) any of the droplets enters another channel, or (3) any droplet exits the microfluidic network, which requires to adapt the equation system (i.e. add, move, or remove the resistance of the droplets) and to resolve it. Overall, by performing this procedure in a loop, the paths of the droplets and, thus, the behavior of a droplet-based microfluidic network can be simulated. This allows to validate whether a network works as intended and even allows to make further design exploration, without an actual fabrication of a prototype.

For example, with the help of this simulator, it was possible to significantly improve the design of a device which is able to screen drug compounds that inhibit the tau-peptide aggregation [4]. The original design of this device took a whole month, required six different prototypes, and produced costs of about $\$ 1200$. Using simulations (rather than a "trial-and-error" approach), these efforts could be reduced to a single day, one prototype, and costs of $\$ 200$ only [15].

Overall, automatic tools can significantly aid engineers in the design and simulation of microfluidic devices. A big challenge remains to make them accessible to the respective stakeholders. First attempts in this regard are provided for the meander design and the simulation in terms of web-interfaces which are available at http:// iic.jku.at/eda/research/meander_designer and http://iic.jku.at/eda/ research/microfluidics_simulation, respectively.

\section{Practical realization}

The design and simulation tools discussed in the previous section are very important for the development of microfluidic chips. However, since these methods are based on the abstract 1D model, it is inevitable to conduct practical experiments after the design process in order to validate the predicted behavior.

Although many works have theoretically investigated the concept of microfluidic networking, it suffers from limited experimental validation. One possible reason for this issue is that this concept requires the precise generation of droplets at prescribed times with certain volumes and at certain distances, so-called droplet-ondemand (DoD). Moreover, it is necessary to generate two different droplet types (header and payload droplet) on a single channel. In the following, we present a simple droplet generation method that fulfills the aforementioned requirements. The simplicity of the proposed methods allows a simple and low cost fabrication method that is also discussed here.

\subsection{Microfluidic chip fabrication}

The microfluidic chips for the experimental results reported below are fabricated using a simple and low-cost method. As material we use PMMA (Polymethylmethacrylate) sheets and the desired microchannel structure is fabricated through laser engraving using a $\mathrm{CO}_{2}$ laser. Then the inlet holes for the fluid supply are drilled. The microfluidic chip is finalized through ethanol-based thermal bonding, which consists of two steps: (i) a thin layer of ethanol is spread between the engraved PMMA layer and a PMMA cover layer; (ii) the two layers are aligned, covered with a thermoconducting, protective membrane and placed in a hot press to achieve final bonding. This prototyping method allows a fast $(\sim 5 \mathrm{~min})$ and cost-effective ${ }^{6}$ $(\$ 0.5)$ fabrication of microfluidic chips, which requires no special skills and environments.

${ }^{6}$ The costs include the required material per chip, i.e., PMMA sheets and ethanol.

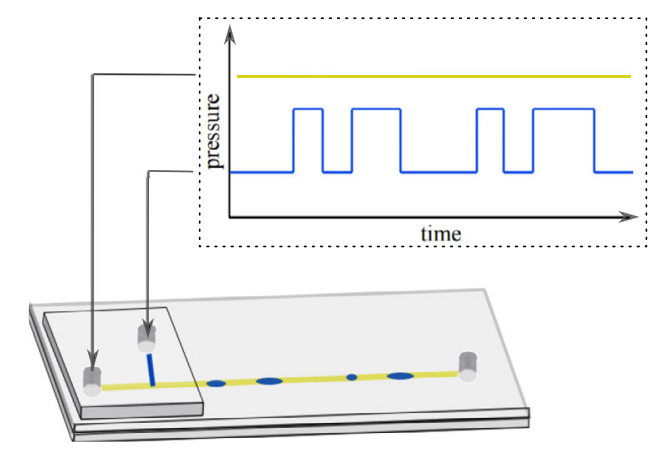

Fig. 6. Droplet-on-demand principle

\subsection{Droplet-on-demand}

For many applications it is crucial to have precise control over the droplet parameters, such as size or distance. Such DoD methods can be either realized using on-chip (e.g., integrated microvalves [1, 38]) or off-chip control of the fluids. Since on-chip methods usually suffer from a complex, multilevel fabrication process various off-chip techniques have been developed [5, 35]. Although, these methods overcome the problems of on-chip methods, they suffer from high synchronization requirements between the pressure control of both phases and they lack in the possibility to connect multiple DoD generators on a single channel.

Thus, we have recently developed a DoD system that overcomes the aforementioned issues [22, 23, 25-27]. A schematic of the proposed method is illustrated in Fig. 6, which includes a microfluidic chip with a T-junction for the droplet generation and the pressure of the dispersed and continuous phase are controlled by an external pressure controller. The principle is as follows: First, the system is brought into equilibrium state, i.e. the pressures of the dispersed and continuous phase are balanced that no droplet is generated. The droplet generation is induced by applying a positive pressure pulse to the dispersed phase, while the pressure of the continuous phase is kept constant. At the end of the pulse duration the droplet breaks up and the systems returns to the equilibrium state. We have observed linear correlation between the duration of the applied pulse and the volume of the generated droplets. This approach enables the generation of droplets of different sizes and inter-droplet distances through varying the pulse duration and the time between consecutive pulses, respectively. Since only the dispersed phase needs to be controlled no synchronization between the two phases is necessary and multiple independent dispersed channels can be connected to a single channel. The latter property is a unique feature compared to state-of-the-art off-chip control methods, which allows, for the first time, to practically validate the concept of microfluidic networking and switching.

For example, ${ }^{7}$ Fig. 7 illustrates different droplet sizes that are generated by pressure pulses with different pulse durations and Fig. 8 shows droplet pairs with different types and with different distances that are generated by two cascaded DoD systems. These results provide the basis for the practical realization of size-based and distancebased switching, respectively (see Sect. 3.1). Hence, the presented DoD method is an important step towards the practical realization of microfluidic networks. In order to achieve this ultimate goal current

${ }^{7}$ For the results reported in Figs. 7 and 8, silicone oil and colored deionized water was used as continuous and dispersed phase, respectively. 


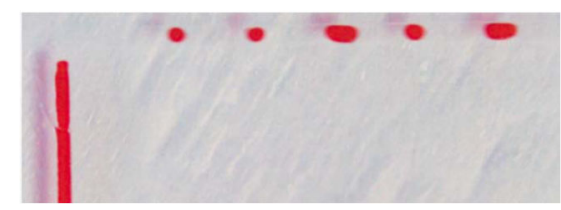

Fig. 7. Generation of droplets of different size, using a single DoD system

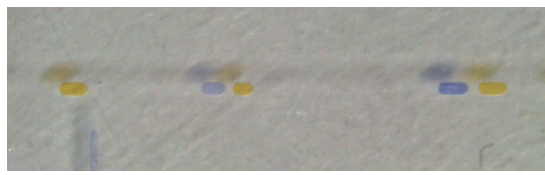

(a)

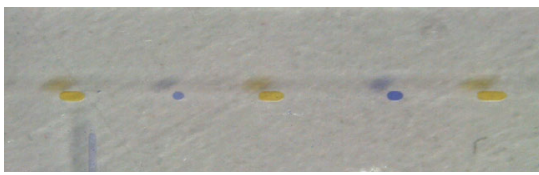

(b)

Fig. 8. Generation of droplet pairs with different types and with (a) small and (b) large distances, using two cascaded DoD systems

research aims to apply this method to single and cascaded microfluidic switches (i.e., microfluidic networks). ${ }^{8}$

\section{Conclusions}

We have provided an overview on the microfluidic networking concepts, which aim to realize flexible and simple lab-on-a-chip devices. In particular, we have presented the theoretical principles and we have introduced design automation methods and simulation tools that support the rather complex design process. Moreover, we have identified the precise generation of droplets as a crucial component for the practical realization of microfluidic networks. Although, we have shown first experimental results on individual components of microfluidic networks (e.g., droplet generation) the practical validation of a complete microfluidic networks is still missing and is part of our current research. Moreover, it would be interesting to further investigate the recently proposed healthcare applications for microfluidic networks, such as drug [28] and pathogen [25] screening.

\section{Acknowledgements}

Open access funding provided by Johannes Kepler University Linz. This work has partially been supported by the LIT Secure and Correct Systems Lab funded by the State of Upper Austria.

Publisher's Note Springer Nature remains neutral with regard to jurisdictional claims in published maps and institutional affiliations.

Open Access Dieser Artikel wird unter der Creative Commons $\mathrm{Na}$ mensnennung 4.0 International Lizenz veröffentlicht, welche die Nutzung, Vervielfältigung, Bearbeitung, Verbreitung und Wiedergabe in jeglichem Medium und Format erlaubt, sofern Sie den/die ursprünglichen Autor(en) und die Quelle ordnungsgemäß nennen, einen Link zur Creative Commons Lizenz beifügen und angeben, ob Änderungen vorgenommen wurden. Die in diesem Artikel enthaltenen Bilder und sonstiges Drittmaterial unterliegen ebenfalls der genannten Creative Commons Lizenz, sofern sich aus der Abbildungslegende

${ }^{8}$ First preliminary results on microfluidic switching can be found here: https:// www.youtube.com/watch?v=qBtG3Vlszkw. nichts anderes ergibt. Sofern das betreffende Material nicht unter der genannten Creative Commons Lizenz steht und die betreffende Handlung nich nach gesetzlichen Vorschriften erlaubt ist, ist für die oben aufgeführten Weiterverwendungen des Materials die Einwilligung des jeweiligen Rechteinhabers einzuholen. Weitere Details zur Lizenz entnehmen Sie bitte der Lizenzinformation auf http://creativecommons.org/licenses/by/4.0/deed.de.

\section{References}

1. Babahosseini, H., Misteli, T., DeVoe, D. L. (2019): Microfluidic on-demand drople generation, storage, retrieval, and merging for single-cell pairing. Lab Chip, 19(3), 493-502.

2. Bruus, H. (2008): Theoretical microfluidics (Vol. 18). Oxford: Oxford University Press.

3. Castorina, G., Reno, M., Galluccio, L., Lombardo, A. (2017): Microfluidic networking: switching multidroplet frames to improve signaling overhead. Nano Commun. Netw., $14,48-59$.

4. Chen, X., Ren, C. L. (2017): A microfluidic chip integrated with droplet generation, pairing, trapping, merging, mixing and releasing. RSC Adv., 7(27), 16738-16750.

5. Churski, K., Nowacki, M., Korczyk, P. M., Garstecki, P. (2013): Simple modular systems for generation of droplets on demand. Lab Chip, 13(18), 3689-3697.

6. Cristobal, G., Benoit, J. P., Joanicot, M., Ajdari, A. (2006): Microfluidic bypass for efficient passive regulation of droplet traffic at a junction. Appl. Phys. Lett., 89(3), 034104

7. De Leo, E., Galluccio, L., Lombardo, A., Morabito, G. (2012): Networked labs-on-a-chip (NLoC): introducing networking technologies in microfluidic systems. Nano Commun Netw., 3(4), 217-228

8. De Leo, E., Donvito, L., Galluccio, L., Lombardo, A., Morabito, G., Zanoli, L. M. (2013): Communications and switching in microfluidic systems: pure hydrodynamic control for networking labs-on-a-chip. IEEE Trans. Commun., 61(11), 4663-4677.

9. Dittrich, P. S., Manz, A. (2006): Lab-on-a-chip: microfluidics in drug discovery. Nat. Rev. Drug Discov., 5(3), 210.

10. Donvito, L., Galluccio, L., Lombardo, A., Morabito, G. (2016): $\mu$-Net: a network for molecular biology applications in microfluidic chips. IEEE/ACM Trans. Netw., 24(4), 2525-2538.

11. Fink, G., Grimmer, A., Hamidović, M., Haselmayr, W., Wille, R. (2020): Robustness analysis for droplet-based microfluidic networks. IEEE Trans. Comput.-Aided Des. Integr. Circuits Syst. https://doi.org/10.1109/TCAD.2019.2962777.

12. Fuerstman, M. J., Lai, A., Thurlow, M. E., Shevkoplyas, S. S., Stone, H. A., Whitesides, G. M. (2007): The pressure drop along rectangular microchannels containing bubbles. Lab Chip, 7(11), 1479-1489.

13. Glatzel, T., Litterst, C., Cupelli, C., Lindemann, T., Moosmann, C., Niekrawietz, R., Streule, W., Zengerle, R., Koltay, P. (2008): Computational fluid dynamics (CFD) software tools for microfluidic applications-a case study. Comput. Fluids, 37(3), 218235.

14. Glawdel, T., Ren, C. L. (2012): Global network design for robust operation of microfluidic droplet generators with pressure-driven flow. Microfluid. Nanofluid., 13(3) $469-480$.

15. Grimmer, A., Chen, X., Hamidović, M., Haselmayr, W., Ren, C. L., Wille, R. (2018): Simulation before fabrication: a case study on the utilization of simulators for the design of droplet microfluidic networks. RSC Adv., 8, 34733-34742.

16. Grimmer, A., Frank, P., Ebner, P., Häfner, S., Richter, A., Wille, R. (2018): Meander designer: automatically generating meander channel designs. Micromachines, 9(12), 625.

17. Grimmer, A., Haselmayr, W., Springer, A., Wille, R. (2018): Design of applicationspecific architectures for networked labs-on-chips. IEEE Trans. Comput.-Aided Des. Integr. Circuits Syst., 37(1), 193-202.

18. Grimmer, A., Hamidović, M., Haselmayr, W., Wille, R. (2019): Advanced simulation of droplet microfluidics. ACM J. Emerg. Technol. Comput. Syst., 15(3), 1-16.

19. Grimmer, A., Haselmayr, W., Wille, R. (2018): Automated dimensioning of Networked Labs-on-Chip. IEEE Trans. Comput.-Aided Des. Integr. Circuits Syst., 38(7), 12161225

20. Grimmer, A., Haselmayr, W., Wille, R. (2020): Automatic droplet sequence generation for microfluidic networks with passive droplet routing. IEEE Trans. Comput.-Aided Des. Integr. Circuits Syst., 39(2), 387-396.

21. Gu, H., Duits, M. H., Mugele, F. (2011): Droplets formation and merging in two-phase flow microfluidics. Int. J. Mol. Sci., 12(4), 2572-2597.

22. Hamidović, M., Haselmayr, W., Grimmer, A., Wille, R. (2018): Droplet-on-demand for realizing flexible and programmable lab-on-chip devices. In Int. conf. Miniaturized systems for chemistry and life sciences (pp. 1-2).

23. Hamidović, M., Haselmayr, W., Grimmer, A., Wille, R. (2018): Towards droplet on demand for microfluidic networks. In Proc. workshop molecular communications (pp. 12).

24. Hamidović, M., Haselmayr, W., Grimmer, A., Wille, R., Springer, A. (2018): Comparison of switching principles in microfluidic bus networks. In Proc. int. conf. nanoscale computing and communications (pp. 1-6). 
25. Hamidović, M., Haselmayr, W., Grimmer, A., Wille, R., Springer, A. (2019): Passive droplet control in microfluidic networks: a survey and new perspectives on their practical realization. Nano Commun. Netw., 19, 33-46.

26. Hamidović, M., Marta, U., Grimmer, A., Fink, G., Wille, R., Bridle, H., Springer, A., Haselmayr, W. (2019): First practical realization of switching in microfluidic networks. In Proc. workshop molecular communications (pp. 1-2)

27. Hamidović, M., Marta, U., Bridle, H., Hamidović, D., Fink, G., Wille, R., Springer, A. Haselmayr, W. (2020): Off-chip controlled droplet-on-demand method for precise sample handling. ACS Omega.

28. Haselmayr, W., Hamidović, M., Grimmer, A., Wille, R. (2018): Fast and flexible drug screening using a pure hydrodynamic droplet control. In Proc. Euro. conf. microfluidics (pp. 1-4)

29. Haselmayr, W., Zanella, A., Morabito, G. (2019): Communications and networking in droplet-based microfluidic systems (pp. 1-7). Cham: Springer.

30. Haselmayr, W., Zanella, A., Morabito, G. (2019): Droplet-based microfluidics: communications and networking. Boca Raton: CRC Press.

Authors

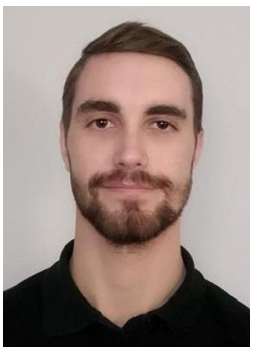

\section{Gerold Fink}

received the Master's degree in mechatronics from the Johannes Kepler University Linz Austria, in 2019. Currently, he is a Ph.D. student at the Institute of Integrated Circuits at the Johannes Kepler University. His research area focuses on simulations and design automations for microfluidic networks.

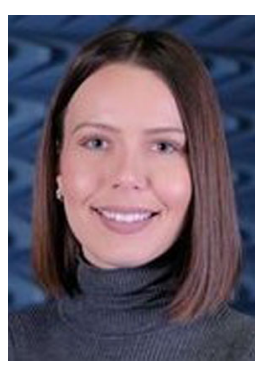

\section{Medina Hamidović}

has received her B.Sc. degree in electrical engineering at the University of Tuzla in 2014. In 2017, Hamidovic received her joint Master's degree in electrical engineering from HeriotWatt University (UK), University of South East Norway and Budapest University of Technology and Economics. At the moment, Hamidovic is a Ph.D. researcher at the Institute for Communications Engineering and RFSystems at the Johannes Kepler University Linz (Austria). Her research is focused on the area of microfluidic networks and networked LoC devices.

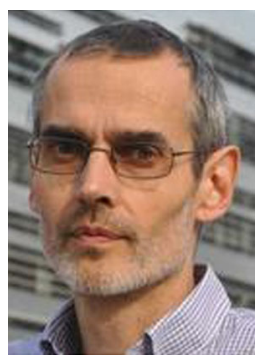

\section{Andreas Springer}

received the Dipl.-Ing. degree in Electrical Engineering from the Technical University of $\mathrm{Vi}$ enna, Austria, in 1991, the Dr. techn. (Ph.D) degree and the Univ.-Doz. (Habilitation) degree both from the Johannes Kepler University Linz (JKU), Austria, in 1996 and 2001, respectively. From 1991 to 1996 he was with the Microelectronics Institute at JKU. In 1997 he joined the Institute for Communications and Information Engineering at the same university, where he became a full professor in 2005. Since July 2002 he is also head of the Institute for Communications Engineering and RF-Systems (formerly Institute for Communications and Information Engineering) at JKU. In the Austrian K2 Center for Symbiotic Mechatronics he serves as a Research Area Coordinator. Since 2017 he is co-leader of the "Christian Doppler Lab for Digitally Assisted RF Transceivers for Future Mobile Communications". He was member of the editorial board of the International Journal of Electronics and Communications from 2012 to 2019, and he serves as reviewer for a number
31. Mark, D., Haeberle, S. (2010): Microfluidic lab-on-a-chip platforms: requirements, characteristics and applications. Chem. Soc. Rev., 39(3), 1153-1182.

32. Oh, K. W., Lee, K., Ahn, B., Furlani, E. P. (2012): Design of pressure-driven microfluidic networks using electric circuit analogy. Lab Chip, 12(3), 515-545.

33. Pollack, M. G., Shenderov, A. D., Fair, R. B. (2002): Electrowetting-based actuation of droplets for integrated microfluidics. Lab Chip, 2(2), 96-101.

34. Teh, S. Y., Lin, R., Hung, L. H., Lee, A. P. (2008): Droplet microfluidics. Lab Chip, 8, 198-220.

35. Teo, A. J. T., Li, K. H. H., Nguyen, N. T., Guo, W., Heere, N., Xi, H. D., Tsao, C. W., Li, W., Tan, S. H. (2017): Negative pressure induced droplet generation in a microfluidic flow-focusing device. Anal. Chem., 89(8), 4387-4391.

36. Wixforth, A. (2003): Acoustically driven planar microfluidics. Superlattices Microstruct., 33(5), 389-396.

37. Zanella, A., Biral, A. (2014): Design and analysis of a microfluidic bus network with bypass channels. In Proc. IEEE int. conf. communications (pp. 3993-3998)

38. Zeng, S., Li, B., Su, X., Qin, J., Lin, B. (2009): Microvalve-actuated precise control of individual droplets in microfluidic devices. Lab Chip, 9(10), 1340-1343. of international journals and conferences. He has been engaged in research work on GaAs integrated millimeter-wave TED's, MMIC's and millimeter-wave sensor systems. His current research interests are focused on wireless communication systems, architectures and algorithms for multi-band/multi-mode transceivers, wireless sensor networks, and recently molecular communications. In these fields, he has published more than 250 papers in journals and at international conferences, one book, and two book chapters. In 2006 he was co-recipient of the science prize of the German Aerospace Center (DLR). Dr. Springer is a member of the IEEE Microwave Theory and Techniques, the Communications, and the Vehicular Technology societies, OVE, and VDI. From 2002 to 2012 he served as Chair of the IEEE Austrian Joint COM/MTT Chapter.

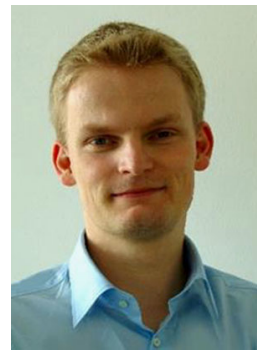

\section{Robert Wille}

(M'06-SM'15) is Full Professor at the Johannes Kepler University Linz, Austria. He received the Diploma and Dr.-Ing. degrees in Computer Science from the University of Bremen, Germany, in 2006 and 2009, respectively. Since then, he worked at the University of Bremen, the German Research Center for Artificial Intelligence (DFKI), the University of Applied Science of Bremen, the University of Potsdam, and the Technical University Dresden. Since 2015, he is working in Linz. His research interests are in the design of circuits and systems for both conventional and emerging technologies. In these areas, he published more than 250 papers in journals and conferences and served in editorial boards and program committees of numerous journals/conferences such as TCAD, ASP-DAC, DAC, DATE, and ICCAD. For his research, he was awarded, e.g., with a Best Paper Award at ICCAD, a DAC Under-40 Innovator Award, a Google Research Award, and more. 


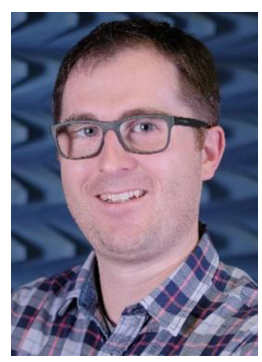

\section{Werner Haselmay}

is an Assistant Professor at the Institute for Communications Engineering and RFSystems, Johannes Kepler University (JKU) Linz, Austria. He received the Ph.D. degree in mechatronics from the same university in 2013. His research interests include the design and analysis of synthetic molecular communication systems and communications and networking in droplet-based microfluidic sys- tems. He has given several invited talks and tutorials on various aspects of droplet-based communications and networking. He has authored 2 book chapters and more than 40 paper, appeared in top-level international peer-reviewed journals and conference proceedings. Moreover, he co-organized the 4th Workshop on Molecular Communications 2019, which was held at JKU Linz. Currently, he serves as Associate Editor for the IEEE Transactions on Molecular, Biological, and Multi-Scale Communications. 